\title{
ON DYNAMICS IN A FOUR-DIMENSIONAL MODEL OF A SMALL OPEN ECONOMY
}

\author{
MÁRIA GRAUSOVÁ ${ }^{\mathrm{a}, *}$, MIROSLAV HUŽVÁR ${ }^{\mathrm{a}}$, RUDOLF ZIMKA ${ }^{\mathrm{a}}$ \\ maria.grausova@umb.sk,miroslav.huzvar@umb.sk,rudolf.zimka@umb.sk \\ a Matej Bel University in Banská Bystrica, Faculty of Economics, Department of Quantitative Methods and \\ Information Systems, Tajovského 10, Banská Bystrica, Slovakia
}

\begin{abstract}
In the paper, a four-dimensional nonlinear macroeconomic model of a small open economy describing the development of net real national income, real physical capital stock, nominal interest rate and nominal money stock is analysed. The functions of investment, savings, real income tax, liquidity preference and net exports that are included in the model are set up in accordance with economic theories. Therefore, the dependence of investments on net real national income is captured through an $S$-shaped curve, and the dependence of investments on nominal interest rate is modelled by an inverse of $S$-shaped curve. The Hopf bifurcation is applied to determine the bifurcation parameter of the model, to formulate the bifurcation equation, and to make a statement on the existence of business cycles.
\end{abstract}

\section{Key words}

macroeconomic dynamic model, equilibrium, stability, center manifold, bifurcation equation, business cycles

\section{JEL classification \\ C61, E32, E37, E52}

\section{Introduction}

The main aim of governments in economic domain is to guarantee sustainable economic growth. In spite of their efforts, undesirable qualitative changes in the development of economies appear and business crises arise, causing big economic damages. Theoretical reasoning for their occurrence was given, for example, by Minsky (1986). According to his financial instability hypothesis, a capitalist economy is inherently unstable. It seems that recent turbulence in the world economy has proved it. But Minsky did not think that such inherent instability was uncontrollable by the government and the central bank. He emphasized that it is important to "stabilize an unstable economy" by means of proper macroeconomic stabilization policies implemented by the government and the central bank. In order to be efficient in these policies, it is important to know how business crises arise and how they can be controlled. Therefore, the study of dynamic nonlinear macroeconomic models may contribute to this domain.

In the paper, we deal with a macroeconomic nonlinear dynamic model of a small open economy consisting of four differential equations which was introduced and analysed by Makovínyiová and Zimka (2009, 2013). This model describes the development of real income, real physical capital stock, nominal interest rate and nominal money stock in a small open economy. It comes out from the classical IS-LM structure and its general form is 


$$
\begin{aligned}
& \dot{Y}=\alpha[I(Y, K, R)+G-S(Y, R)-T(Y)+J(Y, \rho)], \\
& \dot{K}=I(Y, K, R), \\
& \dot{R}=\beta[L(Y, R)-M], \\
& \dot{M}=J(Y, \rho)+\gamma\left(R-R_{f}\right),
\end{aligned}
$$

where $Y$ - net real national income, $K$ - real physical capital stock, $R$ - nominal rate of interest of domestic country, $M$ - nominal money stock, $I$ - net real private investment expenditure on physical capital, $G$ - real government expenditure (fixed), $S$ - savings, $T$ real income tax, $J$ - net exports in real terms, $\rho$ - exchange rate (value of a unit of foreign currency in terms of domestic currency), $L$ - liquidity preference function, $R_{f}$ - nominal rate of interest of foreign country, $\alpha, \beta, \gamma$ - positive parameters, $t$ - time and $\dot{Y}=d Y / d t$, $\dot{K}=d K / d t, \dot{L}=d L / d t, \dot{M}=d M / d t$.

Model (1) expands a three-dimensional Schinasi's model of a closed economy which was introduced and analyzed in Shinasi (1982), as it investigates the case of open economy, and moreover, it describes the development of real physical capital stock.

The model can be also looked at as a generalisation of Asada's three - dimensional model of a small open economy which was introduced and analyzed in Asada (1995). It is assumed in Asada's model that parameter $\beta$ in the equation for interest rate $\dot{R}=\beta[L(Y, R)-M]$ is infinite so that the interest rate adjusts instantaneously to preserve the equilibrium $M=L(Y, R)$ on the money market. This assumption is rather strong and not very realistic. In our paper the equation for the rate of interest is present. It enlarges the dimension of the model which makes it more complex to analyse, but on the other hand, it offers further possibilities for the investigation of variables involved in the model.

For the analysis of model (1) it is useful to express the basic economic properties of the functions involved in the model by equivalent mathematical expressions. We know, for example, that increasing national income $Y$ creates good conditions for the increase of investments $I(Y, K, R)$, and, on the other hand, with increasing interest rate $R$ firms have harder conditions to get money for investments. These facts can also be expressed in words in the following way: the partial derivative of investment $I(Y, K, R)$ with respect to national income $Y$ is positive and the partial derivative of investment $I(Y, K, R)$ with respect to interest rate $R$ is negative. Analogously, we could also describe by mathematical expressions the basic properties of other functions in model (1). These are

$$
\begin{gathered}
\frac{\partial I(Y, K, R)}{\partial Y}>0, \frac{\partial I(Y, K, R)}{\partial K}<0, \frac{\partial I(Y, K, R)}{\partial R}<0, \frac{\partial S(Y, R)}{\partial Y}>0, \frac{\partial S(Y, R)}{\partial R}>0, \\
\frac{\partial T(Y)}{\partial Y}>0, \frac{\partial J(Y, \rho)}{\partial Y}<0, \frac{\partial J(Y, \rho)}{\partial \rho}>0, \frac{\partial L(Y, R)}{\partial Y}>0, \frac{\partial L(Y, R)}{\partial R}<0 .
\end{gathered}
$$

For a deeper study it is necessary to find a proper mathematical expression of individual functions included in the model. Makovínyiová and Zimka $(2009,2013)$ assumed that the investment, savings, liquidity preference and net exports are linear functions with respect to $\mathrm{K}$ and $\mathrm{R}$, and nonlinear functions with respect to $\mathrm{Y}$, but they did not specify these nonlinear functions except a requirement that they are increasing or decreasing. However, functions of very general form are not able to give us suitable results since they also include infinitely many functions that do not have anything common with economically feasible functions, except their monotonicity, having also very big positive but also negative values.

Our goal is to refine and study the model with including such functions of investment, savings and liquidity preference that determine the relationships among individual parameters 
and main variables in accordance with economic theories. Our specific functions are able to describe in a more realistic way the developments of output $Y$, physical capital $K$, interest rate $R$ and money stock $M$ not only in the sense of generally accepted economic theory, but also better describe the real developments of these important macroeconomic variables. Using the specific functions, we will explicitly express the equilibrium point of the model in terms of model parameters and apply Liu's criterion to identify the values of model parameters for which stable or unstable business cycles exist.

The paper is arranged as follows. In Section 2 the functions involved in model (1) are specified. The equilibrium of the model is expressed in Section 3. In Section 4 there are found conditions for model parameters under which Liu's criterion is satisfied. Section 5 is dedicated to the transformations of the model that are necessary to derive the bifurcation equation of the model and formulate the conditions on the existence of limit cycles and their stability/instability. Section 6 summarizes the achieved results and gives suggestions for further research.

\section{Model specification}

In order to gain realistic knowledge on studied economic processes from dynamic models, it is necessary to set them in the way to be able to catch by the functions involved in the model the basic properties of investigated economic processes. Economic theory says (see, e.g., Kaldor, 1940) that the course of the development of investment $I(Y, K, L)$ with respect to $Y$ should have an $S$-shaped form, whereas its development with respect to interest rate $R$ should have the inverse $S$-shaped form. We suggest the function of investment that has exactly these properties with respect to variables $Y$ and $R$. There are also other smaller improvements in our specified functions which are able to describe the reality a little better. For example, the dependence of the investment function on $K$ and the dependence of the liquidity preference function on $R$ are nonlinear in contrast to their linear forms in paper Makovínyiová and Zimka (2009, 2013).

In accordance with previous considerations we specify the functions in model (1) as

$$
\begin{aligned}
& I(Y, K, R)=\frac{\kappa_{1}}{\delta_{1}+e^{-i_{1} Y}}-i_{2} \sqrt{K}+\frac{\kappa_{2}}{\delta_{2}+e^{i_{3} R}}+i_{0}, \\
& S(Y, R)=s_{1} \sqrt{Y-T(Y)}+s_{3} R+s_{0}, \\
& T(Y)=t_{1} Y-t_{0} \\
& L(Y, R)=l_{1} \sqrt{Y}-l_{3} \sqrt{R}+l_{0}, \\
& J(Y)=-j_{1} Y+j_{0},
\end{aligned}
$$

where all constants in these functions are supposed to be positive, $0<t_{1}<1$. 
Putting specified functions (3) into model (1) we receive the model

$$
\begin{aligned}
\dot{Y}= & \alpha\left[\frac{\kappa_{1}}{\delta_{1}+e^{-i_{1} Y}}-i_{2} \sqrt{K}+\frac{\kappa_{2}}{\delta_{2}+e^{i_{3} R}}+i_{0}\right. \\
& \left.+G-s_{1} \sqrt{\left(1-t_{1}\right) Y+t_{0}}-s_{3} R-s_{0}-t_{1} Y+t_{0}-j_{1} Y+j_{0}\right], \\
\dot{K}= & \frac{\kappa_{1}}{\delta_{1}+e^{-i_{1} Y}}-i_{2} \sqrt{K}+\frac{\kappa_{2}}{\delta_{2}+e^{i_{3} R}}+i_{0}, \\
\dot{R}= & \beta\left[l_{1} \sqrt{Y}-l_{3} \sqrt{R}+l_{0}-M\right], \\
\dot{M}= & -j_{1} Y+j_{0}+\gamma\left(R-R_{f}\right) .
\end{aligned}
$$

Further on we will call model (4) simply ,the model“.

\section{Equilibrium of the model}

Equilibria of the model are determined by the system of equations

$$
\begin{aligned}
& \frac{\kappa_{1}}{\delta_{1}+e^{-i_{1} Y}}-i_{2} \sqrt{K}+\frac{\kappa_{2}}{\delta_{2}+e^{i_{3} R}}+i_{0}+G-s_{1} \sqrt{\left(1-t_{1}\right) Y+t_{0}} \\
& -s_{3} R-s_{0}-t_{1} Y+t_{0}-j_{1} Y+j_{0}=0, \\
& \frac{\kappa_{1}}{\delta_{1}+e^{-i_{1} Y}}-i_{2} \sqrt{K}+\frac{\kappa_{2}}{\delta_{2}+e^{i_{3} R}}+i_{0}=0, \\
& l_{1} \sqrt{Y}-l_{3} \sqrt{R}+l_{0}-M=0, \\
& -j_{1} Y+j_{0}+\gamma\left(R-R_{f}\right)=0 .
\end{aligned}
$$

From equation (5.4) we get

$$
R=\frac{1}{\gamma}\left(j_{1} Y-j_{0}\right)+R_{f} .
$$

Putting (6) into equation (5.1) and taking into account equation (5.2), we obtain

$$
G-s_{1} \sqrt{\left(1-t_{1}\right) Y+t_{0}}-\frac{s_{3}}{\gamma}\left(j_{1} Y-j_{0}\right)-s_{3} R_{f}-s_{0}-t_{1} Y+t_{0}-j_{1} Y+j_{0}=0,
$$

and

$$
-\left(\frac{s_{3} j_{1}}{\gamma}+t_{1}+j_{1}\right) Y+G+\frac{s_{3} j_{0}}{\gamma}+t_{0}+j_{0}-s_{3} R_{f}-s_{0}=s_{1} \sqrt{\left(1-t_{1}\right) Y+t_{0}} .
$$

Denoting

$$
a=\frac{s_{3} j_{1}}{\gamma}+t_{1}+j_{1}, \quad b=G+\frac{s_{3} j_{0}}{\gamma}+t_{0}+j_{0}-s_{3} R_{f}-s_{0},
$$

the equation (8) can be written in the form 


$$
-a Y+b=s_{1} \sqrt{\left(1-t_{1}\right) Y+t_{0}} .
$$

The equation (8) has a positive solution if and only if both of the following two conditions hold

$$
\begin{aligned}
& \text { i) }-\left(\frac{s_{3} j_{1}}{\gamma}+t_{1}+j_{1}\right) Y+G+\frac{s_{3} j_{0}}{\gamma}+t_{0}+j_{0}-s_{3} R_{f}-s_{0}>0 \text {, } \\
& \text { ii) } s_{1} \sqrt{t_{0}}<b .
\end{aligned}
$$

From equation (10) we receive

$$
a^{2} Y^{2}-2 a b Y+b^{2}=s_{1}^{2}\left(1-t_{1}\right) Y+s_{1}^{2} t_{0},
$$

and

$$
a^{2} Y^{2}-\left(2 a b+s_{1}^{2}\left(1-t_{1}\right)\right) Y+b^{2}-s_{1}^{2} t_{0}=0 .
$$

Solutions of (13) are given by the formula

$$
Y_{1,2}=\frac{2 a b+s_{1}^{2}\left(1-t_{1}\right) \pm \sqrt{\left(2 a b+s_{1}^{2}\left(1-t_{1}\right)\right)^{2}-4 a^{2}\left(b^{2}-s_{1}^{2} t_{0}\right)}}{2 a^{2}} .
$$

For discriminant of (14) we have

$$
\begin{aligned}
& \left(2 a b+s_{1}^{2}\left(1-t_{1}\right)\right)^{2}-4 a^{2}\left(b^{2}-s_{1}^{2} t_{0}\right)=(2 a b)^{2}+4 a b s_{1}^{2}\left(1-t_{1}\right)+\left(s_{1}^{2}\left(1-t_{1}\right)\right)^{2}-4 a^{2}\left(b^{2}-s_{1}^{2} t_{0}\right) \\
& =4 a b s_{1}^{2}\left(1-t_{1}\right)+\left(s_{1}^{2}\left(1-t_{1}\right)\right)^{2}+4 a^{2} s_{1}^{2} t_{0}>0 .
\end{aligned}
$$

This means, taking into account conditions (11) and the fact that $s_{1} \sqrt{\left(1-t_{1}\right) Y+t_{0}}>0$, that equation (10) has only one solution of the form

$$
Y_{2}=\frac{2 a b+s_{1}^{2}\left(1-t_{1}\right)-\sqrt{\left(2 a b+s_{1}^{2}\left(1-t_{1}\right)\right)^{2}-4 a^{2}\left(b^{2}-s_{1}^{2} t_{0}\right)}}{2 a^{2}} .
$$

Take $Y^{*}=Y_{2}$ as the output's equilibrium value of the model. Substituting $Y$ in (6) by $Y^{*}$ we obtain the equilibrium value of $R$

$$
R^{*}=\frac{1}{\gamma}\left(j_{1} Y^{*}-j_{0}\right)+R_{f} .
$$

From (5.3) we have

$$
M=l_{1} \sqrt{Y}-l_{3} \sqrt{R}+l_{0} .
$$

Substituting $Y$ and $R$ in (18) by their equilibrium values $Y^{*}$ and $R^{*}$, respectively, we get the equilibrium value of $M$

$$
M^{*}=l_{1} \sqrt{Y^{*}}-l_{3} \sqrt{R^{*}}+l_{0} \text {. }
$$


From (5.2) we receive

$$
\sqrt{K}=\frac{1}{i_{2}}\left(\frac{\kappa_{1}}{\delta_{1}+e^{-i_{1} Y}}+\frac{\kappa_{2}}{\delta_{2}+e^{i_{3} R}}+i_{0}\right),
$$

and

$$
K=\frac{1}{\left(i_{2}\right)^{2}}\left(\frac{\kappa_{1}}{\delta_{1}+e^{-i_{1} Y}}+\frac{\kappa_{2}}{\delta_{2}+e^{i_{3} R}}+i_{0}\right)^{2} .
$$

Substituting $Y$ and $R$ in (21) by their equilibrium values $Y^{*}$ and $R^{*}$, respectively, we get the equilibrium value of $K$

$$
K^{*}=\frac{1}{\left(i_{2}\right)^{2}}\left(\frac{\kappa_{1}}{\delta_{1}+e^{-i_{1} Y^{*}}}+\frac{\kappa_{2}}{\delta_{2}+e^{i_{3} R^{*}}}+i_{0}\right)^{2} .
$$

We have shown that there exists an equilibrium point $E=\left(Y^{*}, K^{*}, R^{*}, M^{*}\right)$ of the model. Moreover, for a proper combination of values of model (4) the values of the equilibrium point $E$ are positive, i.e. they comply with their economic sense.

\section{Liu's criterion}

It is known that the occurrence of limit cycles in the dynamic system described by differential equations depends on the existence of proper eigenvalues of the Jacobian matrix at the equilibrium point. We need a pair of purely imaginary eigenvalues with the remaining two with negative real parts. The necessary and sufficient conditions for having such eigenvalues are given by Liu's criterion (Liu, 1994). In accordance with Makovínyiová and Zimka (2013) we find conditions for some parameters of model (4) under which Liu's criterion is satisfied.

The Jacobian matrix of the model at the equilibrium point $E=\left(Y^{*}, K^{*}, R^{*}, M^{*}\right)$ is

$$
J(E)=\left(\begin{array}{cccc}
\alpha F_{11} & \alpha F_{12} & \alpha F_{13} & 0 \\
F_{21} & F_{22} & F_{23} & 0 \\
\beta F_{31} & 0 & \beta F_{33} & -\beta \\
-j_{1} & 0 & \gamma & 0
\end{array}\right),
$$

where

$$
\begin{array}{lrl}
F_{11}=\frac{\kappa_{1} i_{1} e^{-i_{1} Y}}{\left(\delta_{1}+e^{-i_{1} Y}\right)^{2}}-s_{1} \frac{1-t_{1}}{2 \sqrt{\left(1-t_{1}\right) Y+t_{0}}}-t_{1}-j_{1}, F_{21}=\frac{\kappa_{1} i_{1} e^{-i_{1} Y}}{\left(\delta_{1}+e^{-i_{1} Y}\right)^{2}}>0, & F_{31}=\frac{l_{1}}{2 \sqrt{Y}}>0, \\
F_{12}=-\frac{i_{2}}{2 \sqrt{K}}<0, & F_{22}=-\frac{i_{2}}{2 \sqrt{K}}<0, & F_{33}=-\frac{l_{3}}{2 \sqrt{R}}<0 . \\
F_{13}=-\frac{\kappa_{2} i_{3} e^{i_{3} R}}{\left(\delta_{2}+e^{i_{3} R}\right)^{2}}-s_{3}<0, & F_{23}=-\frac{\kappa_{2} i_{3} e^{i_{3} R}}{\left(\delta_{2}+e^{i_{3} R}\right)^{2}}<0,
\end{array}
$$

The characteristic equation of the Jacobian matrix is

$$
\lambda^{4}+a_{1} \lambda^{3}+a_{2} \lambda^{2}+a_{3} \lambda+a_{4}=0 .
$$


Liu's criterion is (see for example Asada, 2003; Liu, 1994)

$$
a_{1}>0, \quad a_{3}>0, \quad a_{4}>0, \quad \Delta_{3}=\left(a_{1} a_{2}-a_{3}\right) a_{3}-a_{1}^{2} a_{4}=0 .
$$

We have

$$
a_{1}=-\left(\alpha F_{11}+F_{22}+F_{33}\right)=-\alpha\left(\frac{\kappa_{1} i_{1} e^{-i_{1} Y}}{\left(\delta_{1}+e^{-i_{1} Y}\right)^{2}}-s_{1} \frac{1-t_{1}}{2 \sqrt{\left(1-t_{1}\right) Y+t_{0}}}-t_{1}-j_{1}\right)+\frac{i_{2}}{2 \sqrt{K}}+\beta \frac{l_{3}}{2 \sqrt{R}} .
$$

Taking into account the signs of entries of Jacobian (23), we see that $a_{1}>0$ for sufficiently large $\beta$ and sufficiently small $\alpha$.

For $a_{2}$ we obtain

$$
\begin{aligned}
a_{2}= & \left|\begin{array}{cc}
\alpha F_{11} & \alpha F_{12} \\
F_{21} & F_{22}
\end{array}\right|+\left|\begin{array}{cc}
\alpha F_{11} & \alpha F_{13} \\
\beta F_{31} & \beta F_{33}
\end{array}\right|+\left|\begin{array}{cc}
\alpha F_{11} & 0 \\
-j_{1} & 0
\end{array}\right|+\left|\begin{array}{cc}
F_{22} & F_{23} \\
0 & \beta F_{33}
\end{array}\right|+\left|\begin{array}{cc}
F_{22} & 0 \\
0 & 0
\end{array}\right| \\
& +\left|\begin{array}{cc}
\beta F_{33} & -\beta \\
\gamma & 0
\end{array}\right|=\alpha\left(F_{11} F_{22}-F_{12} F_{21}\right)+\alpha \beta\left(F_{11} F_{33}-F_{13} F_{31}\right)+\beta F_{22} F_{33}+\beta \gamma .
\end{aligned}
$$

We do not analyze the sign of $a_{2}$ as Liu's criterion does not require it.

The coefficient $a_{3}$ is given by the relation

$$
\begin{aligned}
a_{33}= & -\left\{\begin{array}{ccc}
\alpha F_{11} & \alpha F_{12} & \alpha F_{13} \\
F_{21} & F_{22} & F_{23} \\
\beta F_{31} & 0 & \beta F_{33}
\end{array}|+| \begin{array}{ccc}
\alpha F_{11} & \alpha F_{12} & 0 \\
F_{21} & F_{22} & 0 \\
-j_{1} & 0 & 0
\end{array}|+| \begin{array}{ccc}
F_{22} & F_{23} & 0 \\
0 & \beta F_{33} & -\beta \\
0 & \gamma & 0
\end{array} \mid\right. \\
& \left.+\left|\begin{array}{ccc}
\alpha F_{11} & \alpha F_{13} & 0 \\
\beta F_{31} & \beta F_{33} & -\beta \\
-j_{1} & \gamma & 0
\end{array}\right|\right\}=-\alpha \beta\left(F_{11} F_{22} F_{33}+F_{12} F_{23} F_{31}-F_{13} F_{22} F_{31}-F_{12} F_{21} F_{33}\right) \\
& -\beta \gamma F_{22}-\alpha \beta\left(j_{1} F_{13}+\gamma F_{11}\right),
\end{aligned}
$$

and

$$
a_{3}=-\beta\left[\alpha\left(F_{11} F_{22} F_{33}+F_{12} F_{23} F_{31}-F_{13} F_{22} F_{31}-F_{12} F_{21} F_{33}+j_{1} F_{13}+\gamma F_{11}\right)+\gamma F_{22}\right] .
$$

As the expression $\alpha F_{11}+F_{22}$ can be made negative at sufficiently small $i_{1}$ and large enough $i_{2}$, we get that $a_{3}>0$ at sufficiently small $i_{1}$ and large enough $i_{2}$ and $\gamma$. 
The coefficient $a_{4}$ has the form

$$
\begin{aligned}
a_{4}= & \left|\begin{array}{cccc}
\alpha F_{11} & \alpha F_{12} & \alpha F_{13} & 0 \\
F_{21} & F_{22} & F_{23} & 0 \\
\beta F_{31} & 0 & \beta F_{31} & -\beta \\
-j_{1} & 0 & \gamma & 0
\end{array}\right|=\alpha \beta\left(\gamma F_{11} F_{22}-j_{1} F_{12} F_{23}+j_{1} F_{13} F_{22}-\gamma F_{12} F_{21}\right) \\
& =\alpha \beta\left[\gamma\left(F_{11} F_{22}-F_{12} F_{21}\right)-j_{1}\left(F_{12} F_{23}-F_{13} F_{22}\right)\right]=\alpha \beta \gamma \\
& {\left[\left(\frac{\kappa_{1} i_{1} e^{-i_{1} Y}}{\left(\delta_{1}+e^{-i_{1} Y}\right)^{2}}-s_{1} \frac{1-t_{1}}{2 \sqrt{\left(1-t_{1}\right) Y+t_{0}}}-t_{1}-j_{1}\right)\left(-\frac{i_{2}}{2 \sqrt{K}}\right)-\left(-\frac{i_{2}}{2 \sqrt{K}}\right)\left(\frac{\kappa_{1} i_{1} e^{-i_{1} Y}}{\left(\delta_{1}+e^{-i_{1} Y}\right)^{2}}\right)\right] } \\
& -\alpha \beta j_{1}\left[\left(-\frac{i_{2}}{2 \sqrt{K}}\right)\left(-\frac{\kappa_{2} i_{3} e^{i_{3} R}}{\left(\delta_{2}+e^{i_{3} R}\right)^{2}}\right)-\left(-\frac{\kappa_{2} i_{3} e^{i_{3} R}}{\left(\delta_{2}+e^{i_{3} R}\right)^{2}}-s_{3}\right)\left(-\frac{i_{2}}{2 \sqrt{K}}\right)\right],
\end{aligned}
$$

and

$$
a_{4}=\alpha \beta \gamma\left(s_{1} \frac{1-t_{1}}{2 \sqrt{\left(1-t_{1}\right) Y+t_{0}}}+t_{1}+j_{1}\right)\left(\frac{i_{2}}{2 \sqrt{K}}\right)+\alpha \beta j_{1} s_{3} \frac{i_{2}}{2 \sqrt{K}}
$$

From this we see that $a_{4}>0$ for arbitrary values of $\alpha, \beta$ and $\gamma$.

We have found that the inequalities $a_{1}>0, a_{3}>0$ and $a_{4}>0$ are satisfied at sufficiently small $i_{1}$ and large enough $i_{2}$ and $\gamma$.

Finally, we need to prove that $\Delta_{3}=0$. For this purpose, let us arrange $\Delta_{3}$ as a polynomial with respect to parameter $\beta$. We obtain

$$
\Delta_{3}=g_{0}(N) \beta^{3}+g_{1}(N) \beta^{2}+g_{2}(N) \beta+g_{3}(N),
$$

where symbol $N$ represents all parameters of model (4) except parameter $\beta$. Note that the equilibrium $E=\left(Y^{*}, K^{*}, R^{*}, M^{*}\right)$ is independent on $\beta$.

Suppose that the functions $g_{j}(N), j=0,1,2,3$, are smooth and bounded with respect to all their variables in a domain $\Omega$ on which all expressions in model (4) have economic sense.

Express further the function $g_{0}(N)$ as a polynomial with respect to parameter $\alpha$. We get at $s_{1}=0, \delta_{1}=0, \delta_{2}=0$

$$
g_{0}\left(N\left(s_{1}=0, \delta_{1}=0, \delta_{2}=0\right)\right)=A \alpha^{2}+B \alpha+C,
$$

where 


$$
\begin{aligned}
& A=\frac{l_{3}}{2 \sqrt{R}}\left[-\frac{i_{2} l_{3}^{2} e^{i_{1} Y} i_{1} \kappa_{1}\left(-j_{1}-t_{1}+e^{i_{1} Y} i_{1} \kappa_{1}\right)}{8 \sqrt{K} R}+\frac{i_{2} l_{3}^{2}\left(-j_{1}-t_{1}+e^{i_{1} Y} i_{1} \kappa_{1}\right)^{2}}{8 \sqrt{K} R}\right. \\
& +\frac{l_{3} \gamma\left(-j_{1}-t_{1}+e^{i_{1} Y} i_{1} \kappa_{1}\right)^{2}}{2 \sqrt{R}}+\frac{j_{1} l_{3}\left(-j_{1}-t_{1}+e^{i_{1} Y} i_{1} \kappa_{1}\right)\left(-s_{3}-e^{-i_{3} R} i_{3} \kappa_{2}\right)}{2 \sqrt{R}} \\
& -\frac{i_{2} l_{1} l_{3} e^{i_{1} Y} i_{1} \kappa_{1}\left(-s_{3}-e^{-i_{3} R} i_{3} \kappa_{2}\right)}{8 \sqrt{K} \sqrt{R} \sqrt{Y}}+\frac{2 i_{2} l_{1} l_{3}\left(-j_{1}-t_{1}+e^{i_{1} Y} i_{1} \kappa_{1}\right)\left(-s_{3}-e^{-i_{3} R} i_{3} \kappa_{2}\right)}{8 \sqrt{K} \sqrt{R} \sqrt{Y}} \\
& +\frac{l_{1} \gamma\left(-j_{1}-t_{1}+e^{i_{1} Y} i_{1} \kappa_{1}\right)\left(-s_{3}-e^{-i_{3} R} i_{3} \kappa_{2}\right)}{2 \sqrt{Y}}+\frac{j_{1} l_{1}\left(-s_{3}-e^{-i_{3} R} i_{3} \kappa_{2}\right)^{2}}{2 \sqrt{Y}} \\
& +\frac{i_{2} l_{1}^{2} e^{-i_{3} R} i_{3} \kappa_{2}\left(-s_{3}-e^{-i_{3} R} i_{3} \kappa_{2}\right)}{8 \sqrt{K} Y}+\frac{i_{2} l_{1}^{2}\left(-s_{3}-e^{-i_{3} R} i_{3} \kappa_{2}\right)^{2}}{8 \sqrt{K} Y} \\
& \left.+\frac{i_{2} l_{1} l_{3} e^{-i_{3} R} i_{3} \kappa_{2}\left(-j_{1}-t_{1}+e^{i_{1} Y} i_{1} \kappa_{1}\right)}{8 \sqrt{K} \sqrt{R} \sqrt{Y}}\right] \\
& B=\frac{l_{3}}{2 \sqrt{R}}\left[\frac{i_{2}{ }^{2} l_{3}{ }^{2} e^{i_{1} Y} i_{1} \kappa_{1}}{16 K R}-\frac{i_{2}{ }^{2} l_{3}{ }^{2}\left(-j_{1}-t_{1}+e^{i_{1} Y} i_{1} \kappa_{1}\right)}{16 K R}-\frac{i_{2} l_{3} \gamma\left(-j_{1}-t_{1}+e^{i_{1} Y} i_{1} \kappa_{1}\right)}{2 \sqrt{K} \sqrt{R}}\right. \\
& -\gamma^{2}\left(-j_{1}-t_{1}+e^{i_{1} Y} i_{1} \kappa_{1}\right)+\frac{i_{2} j_{1} l_{3} e^{-i_{3} R} i_{3} \kappa_{2}}{4 \sqrt{K} \sqrt{R}}-\frac{i_{2}^{2} l_{1} l_{3} e^{-i_{3} R} i_{3} \kappa_{2}}{16 K \sqrt{R} \sqrt{Y}} \\
& -\frac{i_{2} l_{1} \gamma e^{-i_{3} R} i_{3} \kappa_{2}}{4 \sqrt{K} \sqrt{Y}}-\frac{i_{2}^{2} l_{1} l_{3}\left(-s_{3}-e^{-i_{3} R} i_{3} \kappa_{2}\right)}{16 K \sqrt{R} \sqrt{Y}} \\
& \left.-j_{1} \gamma\left(-s_{3}-e^{-i_{3} R} i_{3} \kappa_{2}\right)-\frac{i_{2} l_{1} \gamma\left(-s_{3}-e^{-i_{3} R} i_{3} \kappa_{2}\right)}{2 \sqrt{K} \sqrt{Y}}\right] \text {, } \\
& C=\frac{l_{3}}{2 \sqrt{R}}\left(\frac{i_{2}{ }^{2} l_{3} \gamma}{8 K \sqrt{R}}+\frac{i_{2} \gamma^{2}}{2 \sqrt{K}}\right)>0
\end{aligned}
$$

Solving the equation

$$
g_{0}\left(N\left(s_{1}=0, \delta_{1}=0, \delta_{2}=0\right)\right)=A \alpha^{2}+B \alpha+C=0
$$

with respect to parameter $\alpha$ we find conditions for which $g_{0}\left(N\left(s_{1}=0, \delta_{1}=0, \delta_{2}=0\right)\right)=0$. The solutions are given by the formula

$$
\alpha_{1,2}=\frac{-B \pm \sqrt{B^{2}-4 A C}}{2 A} .
$$

We are interested in a positive solution of (39). From (35) we obtain that $A$ will be negative at small enough values of $j_{1}, l_{1}$ and sufficiently large values of $\kappa_{1}$ and $\kappa_{2}$. Then equation (39) has the positive solution 


$$
\alpha_{2}=\frac{-B-\sqrt{B^{2}-4 A C}}{2 A}
$$

We have found that the function $g_{0}(N)$ satisfies the equation $g_{0}(N)=0$ at small enough values of $j_{1}, l_{1}$ and sufficiently large values of $\kappa_{1}, \kappa_{2}, s_{1}=0, \delta_{1}=0, \delta_{2}=0$ and at the value of parameter $\alpha=\alpha_{2}$ which is given by formula (40).

We have shown that there exist such values of parameters of model (4) under which the inequalities $a_{1}>0, a_{3}>0, a_{4}>0$ are satisfied, $g_{0}(N)=0$, and the value of parameter $\alpha=\alpha_{2}$ is given by formula (40). Fix these values and denote them by the symbol $\tilde{N}$.

Put $\sigma=1 / \beta$ and construct in a neighborhood $\Sigma$ of the values $\tilde{N}$ a function

$$
F(\Sigma, \sigma)=\sigma^{3} \Delta_{3} \text {. }
$$

There is

$$
F(\Sigma, \sigma)=\sigma^{3}\left(g_{0} \frac{1}{\sigma^{3}}+g_{1} \frac{1}{\sigma^{2}}+g_{2} \frac{1}{\sigma}+g_{3}\right)
$$

and

$$
F(\Sigma, \sigma)=g_{0}+g_{1} \sigma+g_{2} \sigma^{2}+g_{3} \sigma^{3}
$$

We have

$$
F(\tilde{N}, \sigma=0)=0
$$

and

$$
\frac{\partial F(\Sigma, \sigma)}{\partial \alpha}=\frac{\partial g_{0}(\Sigma, \sigma)}{\partial \alpha}=-\sqrt{b^{2}-4 a c} \neq 0
$$

at the point $\tilde{N}$ and $\sigma=0$. According to Implicit Functional Theorem there exists a function $\alpha=\varphi(\Sigma-\{\alpha\}, \sigma)$ defined in a neighbourhood $\Pi$ of the point $(\tilde{N}-\{\alpha\}, \sigma=0)$ such that $\varphi(\tilde{N}-\{\alpha\}, \sigma=0)=\alpha_{2}$ and $F(\varphi(\Sigma-\{\alpha\}, \sigma), \Sigma-\{\alpha\}, \sigma)=\sigma^{3} \Delta_{3}=0$ on $\Pi$. This means that $\Delta_{3}=0$ on $\Pi$.

By these considerations we have shown that there exist sets of parameters of model (4) under which Liu's criterion is satisfied. Let a symbol $\breve{N}$ represent one of such sets guaranteeing that Liu's criterion is satisfied. Fix all the parameters of $\breve{N}$ except the value $\breve{\alpha}$, and investigate the model in a small neighborhood of $\breve{\alpha}$. We call the value $\breve{\alpha}$ the bifurcation value of the model.

\section{Existence and stability of limit cycles}

In this section we specify the conditions for the existence and stability of limit cycles of model (4). Following Makovínyiová and Zimka (2013), we gradually make three transformations of the model to obtain the form that allows us to derive the bifurcation equation.

First, we perform in model (4) the transformation

$$
x=M u,
$$

where $x=(Y, K, R, M)^{T}, u=(\tilde{Y}, \tilde{K}, \tilde{R}, \tilde{M})^{T},(*)^{T}$ means the transpose vector, and the matrix $M$ consists of the eigenvectors corresponding to the eigenvalues of Jacobian (23) which are arranged in a way to receive the center manifold with respect to variables $\tilde{Y}$ and $\tilde{K}$, and 
move the bifurcation value $\breve{\alpha}$ into the origin by the translation $\tau=\alpha-\breve{\alpha}$. We receive the model with the Jordan linear approximation matrix

$$
\begin{aligned}
& \dot{\tilde{Y}}=\lambda_{1} \tilde{Y}+G_{1}(\tilde{Y}, \tilde{K}, \tilde{R}, \tilde{M}, \tau), \\
& \dot{\tilde{K}}=\lambda_{2} \tilde{K}+G_{2}(\tilde{Y}, \tilde{K}, \tilde{R}, \tilde{M}, \tau), \\
& \dot{\tilde{R}}=\lambda_{3} \tilde{R}+G_{3}(\tilde{Y}, \tilde{K}, \tilde{R}, \tilde{M}, \tau), \\
& \dot{\tilde{M}}=\lambda_{4} \tilde{M}+G_{4}(\tilde{Y}, \tilde{K}, \tilde{R}, \tilde{M}, \tau),
\end{aligned}
$$

where $\lambda_{1}=\mathrm{i} \omega_{0}, \lambda_{2}=-\mathrm{i} \omega_{0}, \operatorname{Re} \lambda_{j}<0, j=3,4, \tilde{K}=\overline{\tilde{Y}}, G_{2}=\bar{G}_{1},(\bar{*})$ means complex conjugation.

Second, we transform model (47) into its partial normal form on the center manifold. Using the polynomial transformation

$$
u=w+h(\hat{Y}, \hat{K}, \tau),
$$

where $u=(\tilde{Y}, \tilde{K}, \tilde{R}, \tilde{M})^{T}, w=(\hat{Y}, \hat{K}, \hat{R}, \hat{M})^{T}, h=\left(h_{1}, h_{2}, h_{3}, h_{4}\right)^{T}$, and

$$
h_{j}(\hat{Y}, \hat{K}, \tau)=\sum_{m_{1}+m_{2}+m \geq 2, m \in\{0,1\}}^{4-2 m} \quad h_{j}^{\left(m_{1}, m_{2}, m\right)} \hat{Y}^{m_{1}} \hat{K}^{m_{2}} \tau^{m}, \quad j=1,2,3,4,
$$

we obtain model

$$
\begin{aligned}
& \dot{\hat{Y}}=\lambda_{1} \hat{Y}+\vartheta_{1} \hat{Y} \tau+\vartheta_{2} \hat{Y}^{2} \hat{K}+U^{0}(\hat{Y}, \hat{K}, \hat{R}, \hat{M}, \tau)+\tilde{U}(\hat{Y}, \hat{K}, \hat{R}, \hat{M}, \tau), \\
& \dot{\hat{K}}=\lambda_{2} \hat{K}+\bar{\vartheta}_{1} \hat{K} \tau+\bar{\vartheta}_{2} \hat{Y} \hat{K}^{2}+\bar{U}^{0}(\hat{Y}, \hat{K}, \hat{R}, \hat{M}, \tau)+\overline{\tilde{U}}(\hat{Y}, \hat{K}, \hat{R}, \hat{M}, \tau), \\
& \dot{\hat{R}}=\lambda_{3} \hat{R}+V_{1}^{0}(\hat{Y}, \hat{K}, \hat{R}, \hat{M}, \tau)+\tilde{V}_{1}(\hat{Y}, \hat{K}, \hat{R}, \hat{M}, \tau), \\
& \dot{\hat{M}}=\lambda_{4} \hat{M}+V_{2}^{0}(\hat{Y}, \hat{K}, \hat{R}, \hat{M}, \tau)+\tilde{V}_{2}(\hat{Y}, \hat{K}, \hat{R}, \hat{M}, \tau),
\end{aligned}
$$

where $(*)^{0}(\hat{Y}, \hat{K}, 0,0, \tau)=0,(\tilde{*})(\sqrt{|\tau|} \hat{Y}, \sqrt{|\tau|} \hat{K}, \sqrt{|\tau|} \hat{R}, \sqrt{|\tau|} \hat{M}, \tau)=O\left((\sqrt{|\tau|})^{5}\right)$.

Resonant terms $\vartheta_{1}$ and $\vartheta_{2}$ in (50) are determined by the formulae

$$
\begin{aligned}
\vartheta_{1}= & \frac{\partial^{2} G_{1}}{\partial \tilde{Y} \partial \tau}, \\
\vartheta_{2}= & \frac{1}{2 \lambda_{2}} \frac{\partial^{2} G_{1}}{\partial \tilde{Y}^{2}} \frac{\partial^{2} G_{1}}{\partial \tilde{Y} \partial \tilde{K}}+\frac{1}{\lambda_{1}} \frac{\partial^{2} G_{1}}{\partial \tilde{Y} \partial \tilde{K}} \frac{\partial^{2} G_{2}}{\partial \tilde{Y} \partial \tilde{K}}+\frac{1}{6 \lambda_{1}} \frac{\partial^{2} G_{1}}{\partial \tilde{K}^{2}} \frac{\partial^{2} G_{2}}{\partial \tilde{Y}^{2}}-\frac{1}{\lambda_{3}} \frac{\partial^{2} G_{1}}{\partial \tilde{Y} \partial \tilde{R}} \frac{\partial^{2} G_{3}}{\partial \tilde{Y} \partial \tilde{K}} \\
& -\frac{1}{\lambda_{4}} \frac{\partial^{2} G_{1}}{\partial \tilde{Y} \partial \tilde{M}} \frac{\partial^{2} G_{4}}{\partial \tilde{Y} \partial \tilde{K}}+\frac{1}{2\left(2 \lambda_{1}-\lambda_{3}\right)} \frac{\partial^{2} G_{1}}{\partial \tilde{K} \partial \tilde{R}} \frac{\partial^{2} G_{3}}{\partial \tilde{Y}^{2}}+\frac{1}{2\left(2 \lambda_{1}-\lambda_{4}\right)} \frac{\partial^{2} G_{1}}{\partial \tilde{K} \partial \tilde{M}} \frac{\partial^{2} G_{4}}{\partial \tilde{Y}^{2}} \\
& +\frac{1}{2} \frac{\partial^{3} G_{1}}{\partial \tilde{Y}^{2} \partial \tilde{K}},
\end{aligned}
$$

while all derivatives are calculated at the values $\hat{Y}=0, \hat{K}=0, \hat{R}=0, \hat{M}=0$, and $\tau=0$.

Third, applying the polar coordinates $\hat{Y}=r e^{i \psi}, \hat{K}=r e^{-i \psi}, \hat{R}=v_{1}, \hat{M}=v_{2}$ to model (50), we get model 


$$
\begin{aligned}
& \dot{r}=r\left(\zeta r^{2}+\eta \tau\right)+P^{0}\left(r, \psi, v_{1}, v_{2}, \tau\right)+\tilde{P}\left(r, \psi, v_{1}, v_{2}, \tau\right), \\
& \dot{\psi}=\omega_{0}+\rho \mu+\varepsilon r^{2}+\frac{1}{r}\left(\Phi^{0}\left(r, \psi, v_{1}, v_{2}, \tau\right)+\tilde{\Phi}\left(r, \psi, v_{1}, v_{2}, \tau\right)\right), \\
& \dot{v}_{1}=\lambda_{3} v_{1}+W_{1}^{0}\left(r, \psi, v_{1}, v_{2}, \tau\right)+\tilde{W}_{1}\left(r, \psi, v_{1}, v_{2}, \tau\right), \\
& \dot{v}_{2}=\lambda_{4} v_{2}+W_{2}^{0}\left(r, \psi, v_{1}, v_{2}, \tau\right)+\tilde{W}_{2}\left(r, \psi, v_{1}, v_{2}, \tau\right),
\end{aligned}
$$

where $\zeta=\operatorname{Re} \vartheta_{2}, \eta=\operatorname{Re} \vartheta_{1}, \rho=\operatorname{Im} \vartheta_{1}, \varepsilon=\operatorname{Im} \vartheta_{2}$.

The equation

$$
\zeta r^{2}+\eta \tau=0
$$

is the bifurcation equation of model (47).

Using the results from the bifurcation theory (see e.g. Bibikov, 1979; Kuznetsov, 2004), we can state the following theorem.

Theorem. Let $\zeta$ and $\eta$ be the coefficients in the bifurcation equation (54). Then it holds:

i) If $\zeta<0$, then there exists in model (47) a stable limit cycle for every small enough $\tau>0$ if $\eta$ is positive, and for every small enough $\tau<0$ if $\eta$ is negative.

ii) If $\zeta>0$, then there exists in model (47) an unstable limit cycle for every small enough $\tau<0$ if $\eta$ is positive, and for every small enough $\tau>0$ if $\eta$ is negative.

\section{Conclusion}

The model studied in this paper describes the development of national income, capital stock, interest rate and money stock and expands a three - dimensional closed economy model of Schinasi (1982) and a four - dimensional open economy model of Makovínyiová and Zimka $(2009,2013)$. In accordance with generally accepted economic theories, it assumes a nonlinear dependence of the investment function on the income, capital stock and the interest rate. Thus, the model brings a more realistic insight to the relations among key macroeconomic variables.

Based on the specified functions included in the model, we expressed its equilibrium point in terms of model parameters. We showed that there exist such values of model parameters under which the Liu's criterion is satisfied, and we found the bifurcation value of the model. Finally, we described the transformations of the model enabling to get the bifurcation equation of the model. The transformations allow us to do the simulations of the dynamic system with real economic data. The analysis of the model results in Theorem which gives sufficient conditions for the existence of stable and unstable limit cycles.

The model analyzed in this paper enables other generalizations of the stated results that were gained by means of the Hopf bifurcation working with one free bifurcation parameter. A generalization of the Hopf bifurcation is the Bautin bifurcation working with two free bifurcation parameters. The Bautin bifurcation gives a more complex picture of the behavior of solutions of the model in a neighborhood of its equilibrium. This is a topic for further research.

\section{Acknowledgements}

The paper was supported by the grant scheme VEGA No. 1/0785/19 "Construction and analysis of dynamic nonlinear models of macroeconomic processes". 


\section{References}

[1] Asada, T. 1995. Kaldorian dynamics in an open economy. In Journal of Economics, 1992, vol. 62, iss. 3, pp. 239-269.

[2] Asada, T., Yoshida, H. 2003. Coefficient criterion for four-dimensional Hopf bifurcations: A complete mathematical characterization and applications to economic dynamics. In Chaos, Solitons and Fractals, 2003, vol. 18, iss. 3, pp. 525-536.

[3] Bibikov, Y. N. 1979. Local theory of nonlinear analytic ordinary differencial equations. Berlin : Springer, 1979. ISBN 3-540-09114-9.

[4] Kaldor, N. 1940. A model of the trade cycle. In Economic Journal, 1940, vol. 50, iss. 197, pp. 69-86.

[5] Kuznetsov, Y. 2004. Elements of applied bifurcation theory. New York : Springer, 2004. ISBN 0-387-21906-4.

[6] Liu, W. M. 1994. Criterion of Hopf bifurcations without using eigenvalues. In Journal of Mathematical Analysis and Applications, 1994, vol. 182, pp. 250-256.

[7] Makovínyiová, K., Zimka, R. 2009. On stability in generalized Schinasi's macroeconomic model under fixed exchange rates. In Tatra Mountains Mathematical Publications, 2009, vol. 43, pp. 115-122.

[8] Makovínyiová, K., Zimka, R. 2013. On the bifurcation of limit cycles in a dynamic model of a small open economy. In European Journal of Applied Mathematics, 2013, iss. 3, vol. 24, pp. 455-470.

[9] Minsky, H. P. 1986. Stabilizing an unstable economy: A twentieth century fund report. London : Yale University Press, 1986, 372 pp. ISBN 0-300-03386-9.

[10] Schinasi, G. J. 1982. Fluctuations in a dynamic, intermediate-run IS-LM model: Applications of the Poincaré-Bendixon theorem. In Journal of Economic Theory, 1982, iss. 2, vol. 28, pp. 369-375. 